\title{
Long-term outcomes of medical therapy versus successful recanalisation for coronary chronic total occlusions in patients with and without type 2 diabetes mellitus
}

Lei Guo ${ }^{1 *+} \mathbb{D}$, Junjie Wang ${ }^{1}$, Huaiyu Ding ${ }^{1}$, Shaoke Meng ${ }^{1}$, Xiaoyan Zhang ${ }^{2}$, Haichen Lv ${ }^{1}$, Lei Zhong ${ }^{1}$, Jian Wu ${ }^{1}$, Jiaying $\mathrm{Xu}^{1}$, Xuchen Zhou ${ }^{1}$ and Rongchong Huang ${ }^{1,3+}$

\begin{abstract}
Background: In this study, we compared the outcomes of medical therapy (MT) with successful percutaneous coronary intervention (PCI) in chronic total occlusions (CTO) patients with and without type 2 diabetes mellitus.

Methods: A total of 2015 patients with CTOs were stratified. Diabetic patients $(n=755,37.5 \%)$ and non-diabetic patients ( $n=1260,62.5 \%$ ) were subjected to medical therapy or successful CTO-PCI. We performed a propensity score matching (PSM) to balance the baseline characteristics. A comparison of the major adverse cardiac events (MACE) was done to evaluate long-term outcomes.

Results: The median follow-up duration was 2.6 years. Through multivariate analysis, the incidence of MACE was significantly higher among diabetic patients compared to the non-diabetic patients (adjusted hazard ratio [HR] 1.32, $95 \%$ confidence interval [CI] 1.09-1.61, $\mathrm{p}=0.005$ ). Among the diabetic group, the rate of MACE (adjusted HR 0.61, $95 \% \mathrm{Cl} 0.42-0.87, \mathrm{p}=0.006$ ) was significantly lower in the successful CTO-PCl group than in the MT group. Besides, in the non-diabetic group, the prevalence of MACE (adjusted HR 0.85, 95\% Cl 0.64-1.15, p=0.294) and cardiac death (adjusted HR 0.94, 95\% Cl 0.51-1.70, $\mathrm{p}=0.825$ ) were comparable between the two groups. Similar results as with the early detection were obtained in propensity-matched diabetic and non-diabetic patients. Notably, there was a significant interaction between diabetic or non-diabetic with the therapeutic strategy on MACE ( $p$ for interaction $=0.036$ ).
\end{abstract}

Conclusions: For treatment of CTO, successful CTO-PCI highly reduces the risk of MACE in diabetic patients when compared with medical therapy. However, this does not apply to non-diabetic patients.

Keywords: Chronic total occlusions, Diabetes, Percutaneous coronary intervention, Medical therapy, Outcomes

\section{Background}

Epidemiology reports have projected that the global number of patients with diabetes mellitus (DM) will

*Correspondence: leiguo@dmu.edu.cn

${ }^{\dagger}$ Lei Guo and Rongchong Huang contributed equally to this work

1 Department of Cardiology, The First Affiliated Hospital of Dalian Medical

University, Dalian, People's Republic of China

Full list of author information is available at the end of the article increase to 360 million by 2030 [1]. Patients with DM experience a greater atherosclerotic burden, higher rate of complex coronary arterial disease (CAD), higher risks of developing postoperative complications, and adverse outcomes after revascularization compared with nondiabetic patients $[2,3]$.

Coronary chronic total occlusions (CTOs) occur in $18-30 \%$ of all diagnostic coronary angiography and pose serious obstacles in the coronary intervention $[4,5]$. Successful percutaneous coronary intervention (PCI) of 
CTOs has been reported to reduce angina, and improve both long-term survival and ventricular function compared to unsuccessful revascularization [6-9]. However, CTO-PCI can be conducted in $10-20 \%$ patients because its procedures are associated with complex lesions, a higher likelihood of procedure failure rates, and risk of major complications compared to the intervention of non-CTO lesions $[4,10,11]$. Therefore, most CTO patients receive medication rather than PCI $[12,13]$.

Furthermore, previous studies have reported that approximately 34 to $40 \%$ of patients with CTOs have DM $[4,14]$. However, there is a paucity of data on whether the clinical outcome of revascularization and medical therapy (MT) differ for diabetic and non-diabetic CTO patients. Moreover, the studies mainly report on outcomes of successful and failed PCI in CTO patients but rarely considered the patients who received MT without CTO-PCI attempt $[15,16]$. Therefore, we sought to compare the clinical outcomes of MT with successful CTOPCI in CTO patients with and without type 2 diabetes mellitus.

\section{Methods Study population}

Coronary angiography was performed in 27,231 consecutively patients at our center from January 2007 to December 2018. Notably, we included 2980 (10.9\%) patients who had at least 1 CTO case. The exclusion criterion was as follows: Patients with ST-segment elevation myocardial infarction (STEMI), have a history of coronary artery bypass grafting (CABG), have type 1 diabetes, underwent failed CTO-PCI or CABG, have a history of cardiogenic shock or had a malignant tumor. After the exclusion, 755 (37.5\%) patients with type $2 \mathrm{DM}$ and $1260(62.5 \%)$ patients without DM were enrolled for the final analysis. Each study group was categorized into 2 groups (successful CTO-PCI or optimal MT) following the initial treatment strategy on an intention-to-treat (ITT) basis (Fig. 1). Patients referred for PCI showed symptomatic angina, and/or myocardial viability in the territory of CTO or inducible ischemia [11, 13]. These were assessed by cardiac magnetic resonance imaging, dimensional echocardiography, or myocardial perfusion scan $[11,13,17]$. Demographic, angiographic, and procedural data were collected by reviewing hospital records and dedicated database. Follow up on the patients was conducted by reviewing hospital readmission records, telephone interviews, or outpatient visits. The patients' personal information was kept confidential. This study was approved by our institutional review board.

\section{Medical treatment and $\mathrm{PCl}$ procedure}

For medical therapy, antiplatelet medication, statins, renin-angiotensin system blockade, $\beta$-blockers, and nitrate were used. Coronary interventions were performed following current standard guidelines. All patients were pre-treated with aspirin and clopidogrel before catheterization. Thereafter, heparin (70-100 IU/ $\mathrm{kg}$ ) was administered before PCI, however, the use of glycoprotein IIb/IIIa inhibitors was at the physician's discretion. Dual-antiplatelet medication was administered to the patients after PCI for at least 12 months.

\section{Study definitions and follow-up}

We defined Diabetes Mellitus as a fasting glucose level $\geq 7.0 \mathrm{mmol} / \mathrm{L}$ or a glucose level $\geq 11.1 \mathrm{mmol} / \mathrm{L}$ at $2 \mathrm{~h}$ after a meal on more than two occasions, or the current use of oral hypoglycemic agents or insulin [18]. Besides, a "CTO lesion" was defined as a complete occlusion with anterograde Thrombolysis In Myocardial Infarction (TIMI) flow grade of 0 for more than 3 months [19]. The duration was determined based on clinical history or previous angiography. Further, we assessed the major adverse cardiac event (MACE) as a "primary endpoint" consisting of cardiac mortality, myocardial infarction (MI), or target vessel revascularization (TVR). The "secondary endpoint" was cardiac mortality. We defined the angiographic success of CTO-PCI as the restoration of TIMI grade 3 flow with residual stenosis of less than $20 \%$ after implanting a drug-eluting stent to the CTO vessel. Cardiac mortality, MI, and TVR were defined as Standardized Definitions [20].

\section{Statistical analysis}

Data for continuous variables were presented as mean \pm standard deviation whereas, data for categorical variables were presented as percentages. The Student's t-test was used to compare differences between groups for continuous variables, whereas Chi-square or the Fisher exact test was used for discrete variables. Kruskal-Wallis test was used to compare non-parametric data. Survival-free of adverse events was determined through Kaplan-Meier analysis and compared using the log-rank test. A multivariable Cox regression model was generated, whereby covariates with either $\mathrm{p}$ values $<0.1$ on the univariate analysis or potential clinically relevant factors including age, sex, smoking, hypertension, dyslipidemia, history of MI, heart failure, chronic kidney disease (CKD), left ventricular ejection fraction (LVEF), left anterior descending artery (LAD) involvement, multivessel disease, Japanese-chronic total occlusion (J-CTO) score, and SYNTAX score were considered as candidate variables. Additionally, we constructed a propensity score matching (PSM) to balance the baseline characteristics 


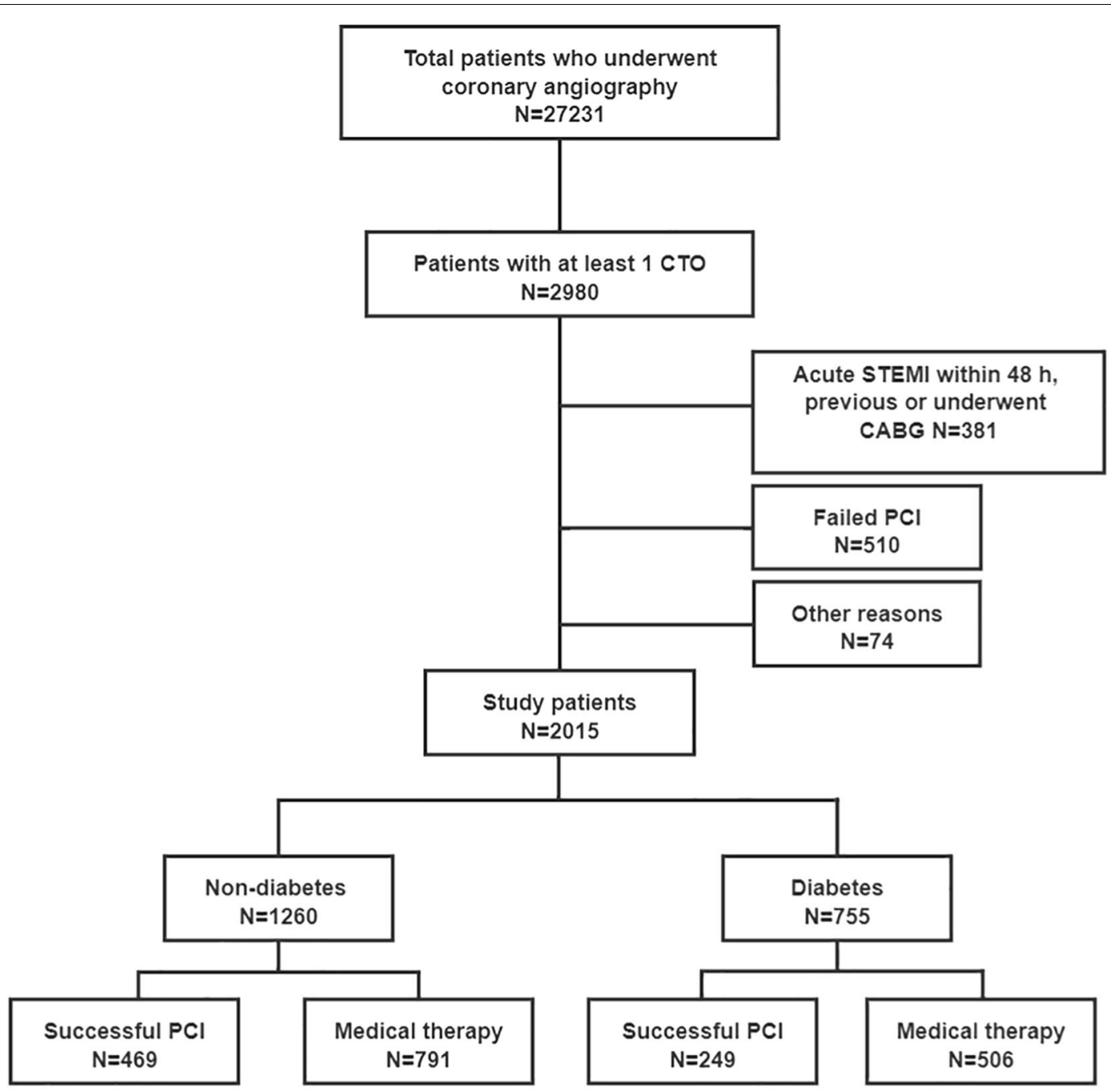

Fig. 1 Study flow chart. CABG coronary artery bypass grafting, CTO chronic total occlusion, PCI percutaneous coronary intervention, STEMI ST-segment elevation myocardial infarction

using the multivariable logistic regression model. The variables used in the PSM are shown in Table 2. The nearest neighbor matching algorithm was used for PSM via a 1: 2 matching protocol. All tests were performed at a 0.05 level. The SPSS Version 24.0 (SPSS Inc., Chicago, Illinois, USA) and Stata Version 15.1 (StataCorp LLC, TX, USA) was used for all statistical computations.

\section{Results}

\section{Characteristics of the study patients}

The prevalence of CTO was $10.9 \%$ in the total population. Notably, out of the 2015 patients with CTOs, 755 (37.5\%) patients had type 2 diabetes whereas, 315 (41.7\%) patients had insulin-dependent DM. The baseline demographic, angiographic, and procedural characteristics of the patients with and without DM are shown in Table 1.
Moreover, patients in the DM group were older and exhibited a higher percentage of hypertension, dyslipidemia, prior MI, CKD and heart failure, more extensive coronary artery disease, lower LVEF, and higher prevalence of female gender than patients in the non-diabetic group. Smoking and CTO of LAD were more common in the non-diabetic group. We did not observe a significant difference in the prevalence of in-hospital death.

In the diabetic group, 506 patients received MT while 249 patients underwent successful CTO-PCI. Notably, patients who underwent successful procedures more often had CTO of LAD and were less likely to develop the multivessel disease, left circumflex coronary artery (LCX) CTO, lesions of calcification, blunt stump, and $\mathrm{J}$-CTO score compared to patients in the MT group. 
Table 1 Baseline clinical, angiographic, and procedural characteristics and in-hospital outcome of all patients with and without diabetes, and of all patients with and without diabetes stratified according to medical therapy or successful CTO-PCI

\begin{tabular}{|c|c|c|c|c|c|c|c|c|c|}
\hline \multirow[t]{2}{*}{ Variables } & \multicolumn{2}{|c|}{ Total population } & \multirow[t]{2}{*}{ P value } & \multicolumn{2}{|c|}{ Patients with diabetes } & \multirow[t]{2}{*}{$P$ value } & \multicolumn{2}{|c|}{ Patients without diabetes } & \multirow[t]{2}{*}{$P$ value } \\
\hline & $\begin{array}{l}\text { Diabetes } \\
(n=755)\end{array}$ & $\begin{array}{l}\text { Non-diabetes } \\
(n=1260)\end{array}$ & & $\begin{array}{l}\text { MT } \\
(n=506)\end{array}$ & $\begin{array}{l}\text { Successful PCI } \\
(n=249)\end{array}$ & & $\begin{array}{l}\text { MT } \\
(n=791)\end{array}$ & $\begin{array}{l}\text { Successful PCI } \\
(n=469)\end{array}$ & \\
\hline Age, years & $65.1 \pm 9.8$ & $63.8 \pm 10.7$ & 0.022 & $65.6 \pm 10.2$ & $64.1 \pm 8.9$ & 0.081 & $64.3 \pm 11.0$ & $62.9 \pm 10.1$ & 0.025 \\
\hline Male & $527(69.8)$ & $1021(81.0)$ & $<0.001$ & $357(70.6)$ & $170(68.3)$ & 0.521 & $650(82.2)$ & $371(79.1)$ & 0.179 \\
\hline Smoking & $275(36.4)$ & $583(46.3)$ & $<0.001$ & $188(37.2)$ & $87(34.9)$ & 0.237 & $364(46.0)$ & $219(46.7)$ & 0.816 \\
\hline Hypertension & $572(75.8)$ & $803(63.7)$ & $<0.001$ & $389(76.9)$ & $183(73.5)$ & 0.308 & $508(64.2)$ & $295(62.9)$ & 0.637 \\
\hline Dyslipidemia & $585(77.5)$ & $900(71.4)$ & 0.015 & $399(78.9)$ & $186(74.7)$ & 0.191 & $565(71.4)$ & $335(71.4)$ & 0.740 \\
\hline Familial history of CAD & $63(8.3)$ & $164(13.0)$ & 0.001 & $44(8.7)$ & $19(7.6)$ & 0.619 & $100(12.6)$ & $41(14.4)$ & 0.609 \\
\hline Previous MI & $309(40.9)$ & $383(30.4)$ & $<0.001$ & $215(42.5)$ & $94(37.8)$ & 0.213 & $271(34.3)$ & $112(23.9)$ & $<0.001$ \\
\hline CKD & $91(12.1)$ & $102(8.1)$ & $<0.001$ & $67(13.2)$ & $24(9.6)$ & 0.138 & $75(9.5)$ & $27(5.8)$ & 0.019 \\
\hline Heart failure & $159(21.1)$ & $181(14.4)$ & 0.004 & $110(21.7)$ & 49 (19.7) & 0.514 & $140(17.7)$ & $41(8.7)$ & $<0.001$ \\
\hline LVEF & $52.0 \pm 10.5$ & $53.2 \pm 9.2$ & 0.010 & $51.4 \pm 11.1$ & $53.3 \pm 9.1$ & 0.320 & $52.3 \pm 9.6$ & $54.8 \pm 8.1$ & $<0.001$ \\
\hline Insulin-dependent DM & $315(41.7)$ & - & - & $219(43.3)$ & $96(38.6)$ & 0.208 & - & - & - \\
\hline \multicolumn{10}{|l|}{ Baseline medication } \\
\hline Aspirin & $727(96.3)$ & $1216(96.5)$ & 0.800 & $484(95.7)$ & $243(97.6)$ & 0.185 & $759(96.0)$ & $457(97.4)$ & 0.165 \\
\hline Clopidogrel & $704(93.2)$ & 1181 (93.7) & 0.668 & $467(92.3)$ & $237(95.2)$ & 0.137 & $723(91.4)$ & $458(97.7)$ & $<0.001$ \\
\hline Statin & $726(96.2)$ & $1205(95.6)$ & 0.337 & $487(96.2)$ & $239(96.0)$ & 0.861 & $756(95.6)$ & $449(95.7)$ & 0.893 \\
\hline$\beta$ blocker & $562(74.4)$ & $957(76.0)$ & 0.445 & $382(75.5)$ & $180(72.3)$ & 0.343 & $591(74.7)$ & $366(78.0)$ & 0.182 \\
\hline ACEl or ARB & $506(67.0)$ & $780(61.9)$ & 0.021 & $358(70.8)$ & $148(59.4)$ & 0.002 & $493(62.3)$ & $287(61.2)$ & 0.689 \\
\hline One CTO lesion & $643(85.2)$ & 1104 (87.6) & 0.116 & $434(85.8)$ & $209(83.9)$ & 0.505 & $697(88.1)$ & 407 (86.8) & 0.486 \\
\hline Two CTO lesions & $106(14.0)$ & $144(11.4)$ & 0.085 & $68(13.4)$ & $38(15.3)$ & 0.498 & $87(11.0)$ & $57(12.2)$ & 0.533 \\
\hline LAD & $237(31.4)$ & $452(35.9)$ & 0.040 & $137(27.1)$ & $100(40.2)$ & $<0.001$ & $262(33.1)$ & $190(40.5)$ & 0.008 \\
\hline LCX & $243(32.2)$ & $348(27.6)$ & 0.029 & $182(36.0)$ & $61(24.5)$ & 0.002 & $243(30.7)$ & $105(22.4)$ & 0.001 \\
\hline RCA & $377(49.9)$ & $604(47.9)$ & 0.385 & $259(51.2)$ & $118(47.4)$ & 0.327 & $379(47.9)$ & $225(48.0)$ & 0.983 \\
\hline Multivessel disease & $630(83.4)$ & $981(77.9)$ & 0.002 & $437(86.4)$ & $193(77.7)$ & 0.002 & $683(86.3)$ & $298(63.5)$ & $<0.001$ \\
\hline Proximal or mid & $514(68.1)$ & $909(72.1)$ & 0.053 & $336(66.4)$ & $187(71.5)$ & 0.159 & $554(70.0)$ & $355(75.7)$ & 0.030 \\
\hline \multicolumn{10}{|l|}{ CTO location } \\
\hline Blunt stump & $313(41.5)$ & $556(44.1)$ & 0.241 & $259(51.2)$ & $54(21.7)$ & $<0.001$ & $408(51.6)$ & $148(31.6)$ & $<0.001$ \\
\hline Calcification & $141(18.7)$ & $220(17.5)$ & 0.491 & $109(21.5)$ & $32(12.9)$ & 0.004 & $158(20.0)$ & $63(13.2)$ & 0.002 \\
\hline Bending $>45^{\circ}$ & $337(44.6)$ & $549(43.6)$ & 0.641 & $221(43.7)$ & $116(46.9)$ & 0.449 & $363(45.9)$ & $186(39.7)$ & 0.031 \\
\hline Length $\geq 20 \mathrm{~mm}$ & $477(63.2)$ & $810(64.3)$ & 0.617 & $320(63.2)$ & $157(63.1)$ & 0.960 & $505(63.8)$ & $305(65.0)$ & 0.670 \\
\hline J-CTO score & $1.66 \pm 1.16$ & $1.67 \pm 1.17$ & 0.930 & $1.78 \pm 1.23$ & $1.41 \pm 0.98$ & $<0.001$ & $1.80 \pm 1.24$ & $1.47 \pm 1.03$ & $<0.001$ \\
\hline SYNTAX score & $23.6 \pm 8.7$ & $21.1 \pm 8.3$ & 0.044 & $24.6 \pm 9.1$ & $21.5 \pm 7.5$ & 0.308 & $22.6 \pm 8.8$ & $18.9 \pm 6.9$ & 0.003 \\
\hline Number of stents & - & - & - & - & $1.46 \pm 0.76$ & - & - & $1.92 \pm 0.99$ & - \\
\hline Total stent length, mm & - & - & - & - & $42.1 \pm 23.1$ & - & - & $23.8 \pm 24.2$ & - \\
\hline Contrast volume, $\mathrm{ml}$ & $175 \pm 76$ & $179 \pm 85$ & 0.844 & $150 \pm 64$ & $228 \pm 72$ & $<0.001$ & $151 \pm 74$ & $226 \pm 83$ & $<0.001$ \\
\hline Coronary dissection & - & - & - & - & $11(4.4)$ & - & - & $15(3.2)$ & - \\
\hline Coronary perforation & - & - & - & - & $2(0.8)$ & - & - & $6(1.3)$ & - \\
\hline In-hospital death & $5(0.7)$ & $5(0.4)$ & 0.622 & - & - & - & - & - & - \\
\hline
\end{tabular}

Values are presented as the mean \pm standard deviation or $\mathrm{n}(\%)$

$A C E l$ angiotensin-converting enzyme inhibitor, $A R B$ angiotensin-receptor blocker, $C A D$ coronary artery disease, $C K D$ chronic kidney disease, $C T O$ chronic total occlusion, DM diabetes mellitus, J-CTO Japanese-chronic total occlusion, LAD left ascending coronary artery, LCX left circumflex coronary artery, LVEF left ventricular ejection fraction, $M I$ myocardial infarction, $M T$ medical therapy, $P C I$ percutaneous coronary intervention, $R C A$ right coronary artery

In the non-diabetic group, 469 patients underwent successful CTO-PCI while 791 patients received MT. Patients who underwent successful CTO-PCI were younger and showed fewer cases of previous MI, CKD, and heart failure, but higher LVEF compared to patients in the MT group. Regarding angiographic characteristics, 
successful CTO-PCI group exhibited fewer cases of multivessel disease, LCX CTO, bending $>45^{\circ}$, calcification, blunt stump, high J-CTO score, and SYNTAX score than the MT group. However, LAD CTO and proximal or mid-CTO locations were more common among patients with successful CTO-PCI arm procedure.

Moreover, in the diabetic group, 270 patients were subjected to MT while 135 patients underwent successful CTO-PCI after PSM. Of note, baseline characteristics were not significantly different between both matched groups. Besides, in the non-diabetic group, 464 patients received MT while 232 patients underwent successful CTO-PCI. Similarly, we did not find considerable differences in the baseline clinical and lesion characteristics among the two matched groups, except for multivessel disease (Table 2).

\section{Follow-up outcomes}

The median follow-up time was 2.6 (interquartile range (IQR), 1.2-4.7) years. Through multivariate analysis,

Table 2 Baseline clinical, angiographic and procedural characteristics of propensity-matched patients with and without diabetes stratified according to medical therapy or successful CTO-PCI

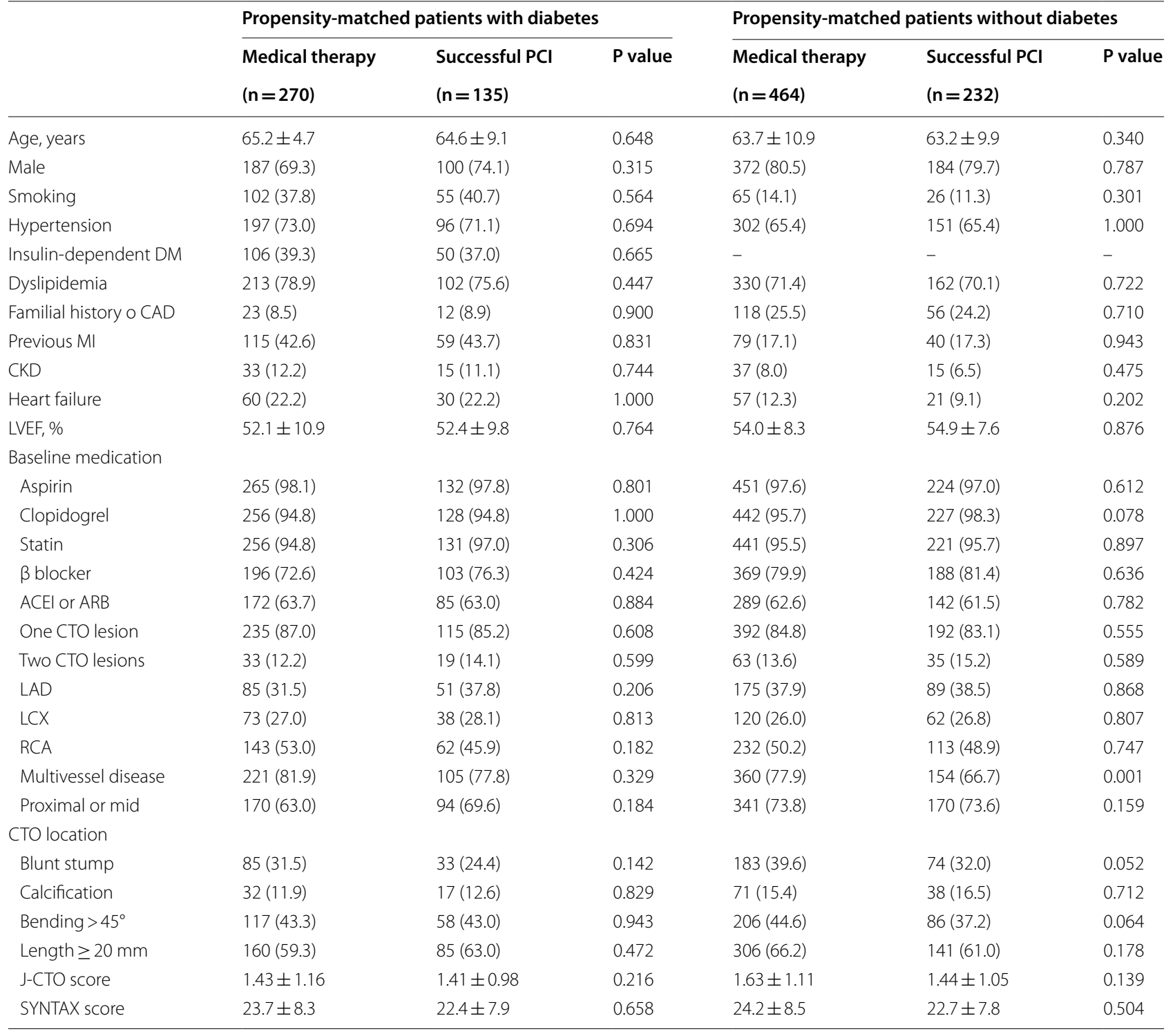

Values are presented as the mean \pm standard deviation or $\mathrm{n}(\%)$

$A C E l$ angiotensin-converting enzyme inhibitor, $A R B$ angiotensin-receptor blocker, $C A D$ coronary artery disease, $C K D$ chronic kidney disease, $C T O$ chronic total occlusion, J-CTO Japanese-chronic total occlusion, $L A D$ left ascending coronary artery, LCX left circumflex coronary artery, LVEF left ventricular ejection fraction, $M I$ myocardial infarction, $\mathrm{PCl}$ percutaneous coronary intervention, $R C A$ right coronary artery 
we found that the MACE rate was significantly higher in the diabetic patients compared to the non-diabetic patients (diabetes vs. non-diabetes: $25.4 \%$ vs. $20.4 \%$, adjusted hazard ratio [HR] 1.32, 95\% confidence interval $[\mathrm{CI}] 1.09-1.61, \mathrm{p}=0.005)$, however, the occurrence of cardiac death (diabetes vs. non-diabetes: $5.0 \%$ vs. $4.7 \%$, adjusted HR $1.13,95 \%$ CI $0.73-1.75, \mathrm{p}=0.597$ ) was not significantly different between the diabetic and non-diabetic groups. In the diabetic group, the incidence of MACE (successful CTO-PCI vs. MT: $18.5 \%$ vs. $28.9 \%$, adjusted HR $0.61,95 \%$ CI $0.42-0.87$, $\mathrm{p}=0.006$ ) and cardiac mortality (successful CTO-PCI vs. MT: $1.4 \%$ vs. $3.7 \%$, adjusted HR 0.29 , $95 \%$ CI $0.10-$ $0.80, p=0.017$ ) were significantly lower in successful
CTO-PCI group compared to the MT group. In the non-diabetic group, the prevalence of MACE (successful CTO-PCI vs. MT: $16.2 \%$ vs. $22.8 \%$, adjusted HR $0.85,95 \%$ CI $0.64-1.15, \mathrm{p}=0.294$ ) and cardiac mortality (successful CTO-PCI vs. MT: $3.8 \%$ vs. $5.2 \%$, adjusted HR $0.94,95 \%$ CI $0.51-1.70, p=0.825$ ) were not significantly different between the 2 groups (Table 3, Fig. 2).

In propensity-matched diabetic patients, those who received MT exhibited a higher rate of MACE (HR $0.54,95 \%$ CI $0.35-0.84, \mathrm{p}=0.006$ ) compared to patients in the successful CTO-PCI group, whereas the incidence of cardiac death (HR 0.334, 95\% CI 0.10-1.17, $\mathrm{p}=0.088$ ) was similar between the two groups. In propensity-matched non-diabetic patients, the rate of

Table 3 Clinical outcomes of all patients with and without diabetes, and of all patients with and without diabetes stratified according to medical therapy or successful CTO-PCI

\begin{tabular}{|c|c|c|c|}
\hline Total population & $\begin{array}{l}\text { Non-diabetes } \\
(n=1260)\end{array}$ & $\begin{array}{l}\text { Diabetes } \\
(n=755)\end{array}$ & P value \\
\hline Cardiac death & $59(4.7)$ & $38(5.0)$ & \\
\hline Adjusted HR (95\% Cl) & 1 & $1.13(0.73-1.75)$ & 0.597 \\
\hline $\mathrm{Ml}$ & $86(6.8)$ & $69(9.1)$ & \\
\hline Adjusted HR (95\% Cl) & 1 & $1.38(0.98-1.92)$ & 0.058 \\
\hline TVR & $155(12.3)$ & $113(15.0)$ & \\
\hline Adjusted HR (95\% Cl) & 1 & $1.41(1.10-1.81)$ & 0.006 \\
\hline MACE & $257(20.4)$ & $192(25.4)$ & \\
\hline Adjusted HR (95\% Cl) & 1 & $1.32(1.09-1.61)$ & 0.005 \\
\hline Patients with diabetes & $\begin{array}{l}\text { Medical therapy } \\
(n=506)\end{array}$ & $\begin{array}{l}\text { Successful PCI } \\
(n=249)\end{array}$ & P value \\
\hline Cardiac death & $33(6.5)$ & $5(2.0)$ & \\
\hline Adjusted HR (95\% Cl) & 1 & $0.29(0.10-0.80)$ & 0.017 \\
\hline $\mathrm{Ml}$ & $50(9.9)$ & $19(7.6)$ & \\
\hline Adjusted HR (95\% Cl) & 1 & $0.75(0.44-1.30)$ & 0.305 \\
\hline TVR & 79 (15.6) & $34(13.7)$ & \\
\hline Adjusted HR (95\% Cl) & 1 & $0.76(0.50-1.17)$ & 0.212 \\
\hline MACE & $146(28.9)$ & $46(18.5)$ & \\
\hline Adjusted HR (95\% Cl) & 1 & $0.61(0.42-0.87)$ & 0.006 \\
\hline Patients with diabetes & $\begin{array}{l}\text { Medical therapy } \\
(n=791)\end{array}$ & $\begin{array}{l}\text { Successful PCI } \\
(n=469)\end{array}$ & P value \\
\hline Cardiac death & $41(5.2)$ & $18(3.8)$ & \\
\hline Adjusted HR (95\% Cl) & 1 & $0.94(0.51-1.70)$ & 0.825 \\
\hline $\mathrm{Ml}$ & $56(7.1)$ & $30(6.4)$ & \\
\hline Adjusted HR (95\% Cl) & 1 & $1.01(0.62-1.65)$ & 0.959 \\
\hline TVR & $109(13.8)$ & $46(9.8)$ & \\
\hline Adjusted HR (95\% Cl) & 1 & $0.85(0.59-1.22)$ & 0.389 \\
\hline MACE & $180(22.8)$ & $77(16.4)$ & \\
\hline Adjusted HR (95\% CI) & 1 & $0.85(0.64-1.15)$ & 0.294 \\
\hline
\end{tabular}

Values are presented as $\mathrm{n}(\%)$

$C l$ confidence interval(s), HR hazard ratio, MACE major adverse cardiovascular events, $M I$ myocardial infarction, $P C l$ percutaneous coronary intervention, $T V R$ targetvessel revascularization 
a

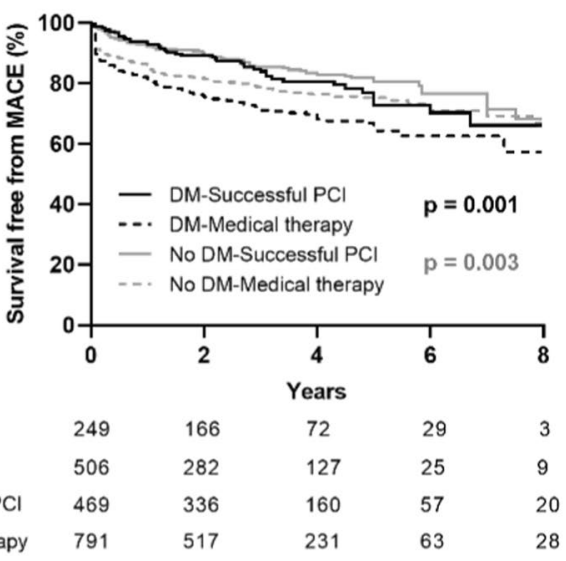

b

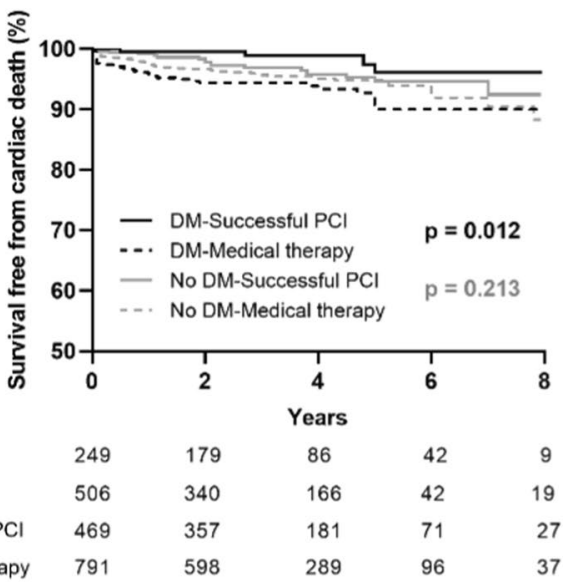

No. at risk

DM Successful PCl DM Medical therapy Non DM Successful PCI Non DM Medical therapy
No. at risk

DM-Successful PCI DM-Medical therapy Non DM-Successful PC Non DM-Medical therapy

Fig. 2 Kaplan-Meier curves for MACE (a) and cardiac death (b) during follow-up for successful CTO-PCI versus medical therapy in total patients with and without diabetes. CTO chronic total occlusion, DM diabetes mellitus, MACE major adverse cardiovascular events, PCI percutaneous coronary intervention

Table 4 Clinical outcomes of propensity-matched patients with and without diabetes stratified according to medical therapy or successful CTO-PCI

\begin{tabular}{|c|c|c|c|}
\hline Patients with diabetes & $\begin{array}{l}\text { Medical therapy } \\
(n=270)\end{array}$ & $\begin{array}{l}\text { Successful PCI } \\
(n=135)\end{array}$ & $P$ value \\
\hline Cardiac death & $17(6.3)$ & $3(2.2)$ & \\
\hline HR $(95 \% \mathrm{Cl})$ & 1 & $0.34(0.10-1.17)$ & 0.088 \\
\hline Ml & $27(10.0)$ & $12(8.9)$ & \\
\hline HR $(95 \% \mathrm{Cl})$ & 1 & $0.88(0.45-1.75)$ & 0.723 \\
\hline TVR & $48(17.8)$ & $19(14.1)$ & \\
\hline HR $(95 \% \mathrm{Cl})$ & 1 & $0.71(0.42-1.20)$ & 0.202 \\
\hline MACE & $86(31.9)$ & $26(19.3)$ & \\
\hline $\mathrm{HR}(95 \% \mathrm{Cl})$ & 1 & $0.54(0.35-0.84)$ & 0.006 \\
\hline Patients without diabetes & $\begin{array}{l}\text { Medical therapy } \\
(n=462)\end{array}$ & $\begin{array}{l}\text { Successful PCI } \\
(n=231)\end{array}$ & $P$ value \\
\hline Cardiac death & $20(4.3)$ & $11(4.8)$ & \\
\hline HR $(95 \% \mathrm{Cl})$ & 1 & $1.05(0.50-2.19)$ & 0.895 \\
\hline $\mathrm{Ml}$ & $33(7.1)$ & $15(6.5)$ & \\
\hline $\mathrm{HR}(95 \% \mathrm{Cl})$ & 1 & $0.91(0.49-1.68)$ & 0.763 \\
\hline TVR & $55(11.9)$ & $23(10.0)$ & \\
\hline HR $(95 \% \mathrm{Cl})$ & 1 & $0.81(0.50-1.32)$ & 0.400 \\
\hline MACE & $90(19.5)$ & $38(16.5)$ & \\
\hline $\mathrm{HR}(95 \% \mathrm{Cl})$ & 1 & $0.80(0.55-1.17)$ & 0.257 \\
\hline
\end{tabular}

Values are presented as $\mathrm{n}(\%)$

$C l$ confidence interval(s), HR hazard ratio, MACE major adverse cardiovascular events, $M I$ myocardial infarction, $P C l$ percutaneous coronary intervention, $T V R$ targetvessel revascularization

MACE (HR 0.80, 95\% CI 0.55-1.17, $\mathrm{p}=0.257$ ) and cardiac mortality (HR 1.05, 95\% CI $0.50-2.19, \mathrm{p}=0.895$ ) were not significantly different in the two groups (Table 4, Fig. 3).
An intention to treat analysis

The baseline characteristics of medical therapy compared with initial CTO-PCI in patients with and without DM are highlighted in Additional file 1: Table S1. Notably, there were 440 diabetic patients and 788 non-diabetic 
a

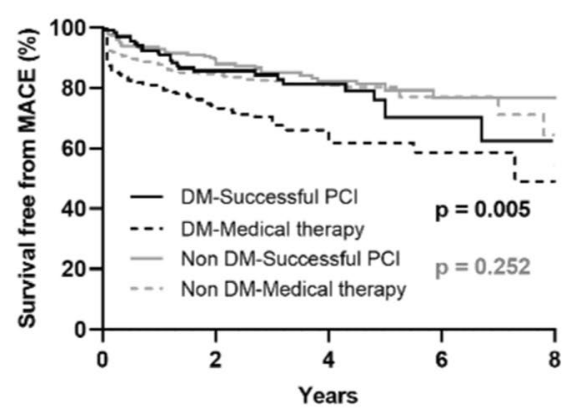

No. at risk

DM-Successful PCI DM-Medical therapy Non DM-Successful PCI Non DM-Medical therapy
$135 \quad 87$

270

231

462
150

169

309

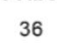

63

83

151
15

14

31

35 b

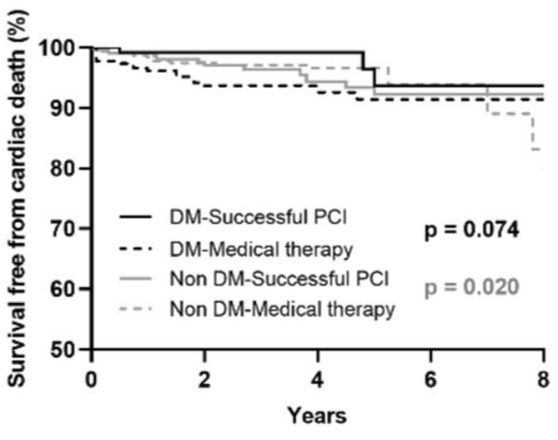

No. at risk

DM-Successful PCI DM-Medical therapy Non DM-Successful PCl Non DM-Medical therapy 462

$\begin{array}{cccc}97 & 45 & 23 & 6 \\ 187 & 88 & 26 & 11 \\ 181 & 94 & 39 & 14 \\ 352 & 185 & 51 & 26\end{array}$

Fig. 3 Kaplan-Meier curves for MACE (a) and cardiac death (b) during follow-up for successful CTO-PCI versus medical therapy in propensity-matched patients with and without diabetes. CTO chronic total occlusion, DM diabetes mellitus, MACE major adverse cardiovascular events, $P C /$ percutaneous coronary intervention

patients who underwent CTO-PCI respectively following ITT. By considering the outcome of MACE, we found that initial CTO-PCI was highly beneficial to diabetic patients (adjusted HR 0.56, 95\% CI 0.42-0.74, p<0.001) compared with MT, however, it was not beneficial to the non-diabetic patients (adjusted HR 0.81, 95\% CI 0.52$1.23, \mathrm{p}=0.297$ ). Cases of cardiac death between diabetic (adjusted HR 0.69, 95\% CI 0.37-1.28, p=0.217) and nondiabetic patients (adjusted HR 1.05, 95\% CI 0.50-2.19, $\mathrm{p}=0.895$ ) were not statistically significant (Additional file 1: Table S2, Fig. 4).

\section{Subgroup analysis}

Further, we noted a significant interaction between diabetic or non-diabetic conditions with therapeutic strategy following MACE $(p=0.036)$. The survival free from MACE benefit of successful CTO-PCI was highly significant among diabetic patients than in non-diabetic patients. Similarly, patients without cases of heart failure benefited from successful CTOPCI for MACE, however, the effect was not observed among the patients with heart failure ( $\mathrm{p}$ for interaction $=0.279$ ). Additionally, the survival free from MACE benefit of successful CTO-PCI was comparable in insulin-dependent DM and non-insulin-dependent

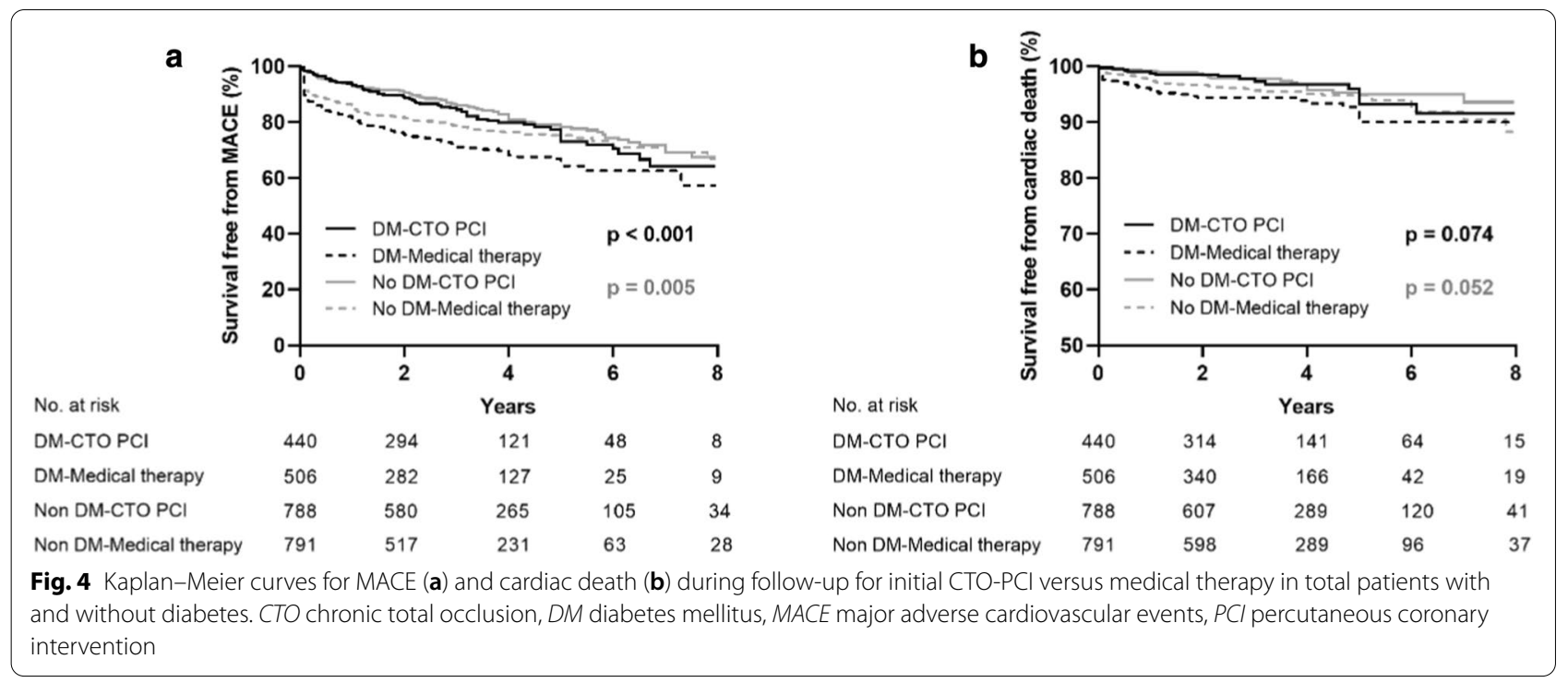




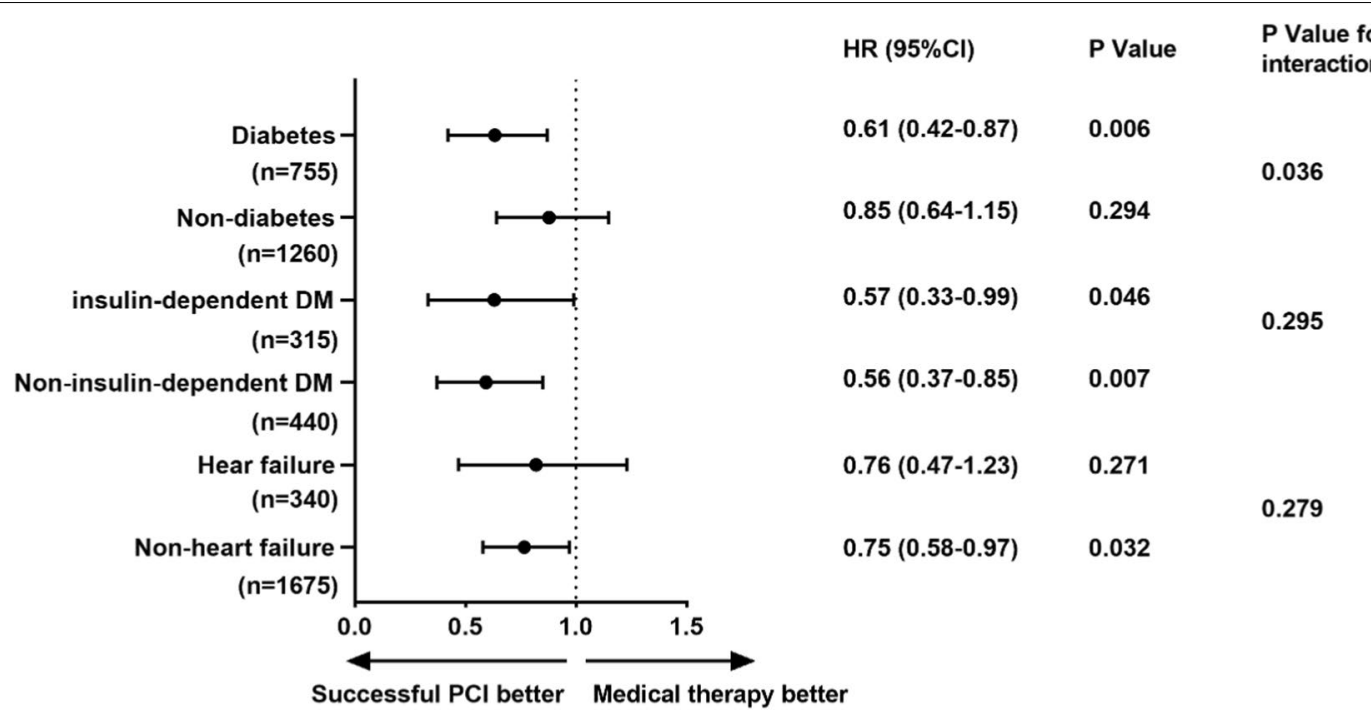

Fig. 5 DM, insulin-dependent DM and heart failure subgroup analysis for MACE. Cl confidence interval(s), DM diabetes mellitus, HR hazard ratio, MACE major adverse cardiovascular events, $\mathrm{PCl}$ percutaneous coronary intervention

DM patients. Besides, the interaction between insulindependent DM or insulin-independent DM with therapeutic strategy following MACE was not significant $(\mathrm{p}=0.295)$, (Fig. 5).

\section{Discussion}

We assessed the long-term outcomes of different treatment strategies in CTO patients with and without type 2 DM in a large cohort population. Notably, we confirmed the following: (1) Diabetic patients with CTOs are highly prone to lower LVEF, multivessel disease, and complex lesions, and encounter more long-term adverse clinical outcomes compared to non-diabetic patients. (2) Successful CTO-PCI reduces MACE compared to the use of medical therapy alone in diabetic patients, this was confirmed by both the multivariable Cox regression and PSM analyses. (3) Successful CTO-PCI is not associated with reduced MACE or cardiovascular mortality in nondiabetic patients with CTOs.

In addition, $\mathrm{DM}$ is an independent risk factor for $\mathrm{CAD}$ and has been reported to be associated with longer coronary lesions, more complex anatomy, comorbidities, and more adverse cardiovascular events [21]. Similarly, we observed that diabetic patients have a significantly higher prevalence of multivessel disease and SYNTAX score compared to non-diabetic patients, this concurs with the findings by Choi and the team [22]. A previous study showed that diabetic patients receiving primary PCI had more common CTO lesions in non-infarct related compared with non-diabetic patients (21\% vs. $12 \%)$ [23]. Moreover, large contemporary CTO registries have reported that $41-45 \%$ of patients undergoing CTO-PCI had DM [24, 25]. However, there are no available reports on the association of the outcomes with an optimal therapeutic strategy in DM or non-DM CTO patients. To our knowledge, this is the first report that assessed the longterm clinical outcomes of successful PCI compared with medical therapy in unselected on a large cohort of CTO patients with and without type 2 diabetes.

Besides, previous studies indicate that DM is an independent risk factor for restenosis, need for revascularization and MACEs, particularly in patients with longer coronary lesion [26, 27]. Additionally, Kandzari et al. demonstrated that diabetic patients with CTOs who underwent PCI with sirolimus-eluting stents exhibited higher rates of restenosis and TVR compared to non-diabetic patients (22\% vs. $4.7 \%$ ) [28], this observation is consistent with our findings. Elsewhere, Safley et al. reported similar survival rates between CTO and non-CTO diabetic patients $(75 \%$ vs. $79 \%, p=0.20)$ after 5 years of follow-up [29]. In the sub-analysis of CIBELES trial whereby 207 patients underwent successful CTO-PCI with a drugeluting stent, the rates of cardiac events including death, MI, and TVR were comparable in diabetic and non-diabetic patients. However, this study enrolled only 75 diabetic patients with $21 \%$ insulin-dependent diabetics and the follow-up period was relatively short (12 months). This may not accurately reflect the clinical outcomes in diabetic and non-diabetic patients [3]. Contrarily, Claessen et al. reported that CTO patients with DM exhibited a higher long-term mortality rate compared to patients without DM [30]. Further, among the diabetic patients, 
successful CTO-PCI was associated with reduced longterm mortality and subsequent CABG [30]. Moreover, a large-scale study that included 6320 patients who underwent PCI showed that mortality is higher in diabetic patients than in non-diabetic patients [31]. In recent a meta-analysis which included 4571 patients with CTO (1915 diabetic patients and 2656 non-diabetic patients), CTO patients with DM exhibited significantly higher rates of mortality, repeated revascularization, and MACEs compared to patients without DM [32]. Likewise, our study reported that TVR and MACE rates are higher in diabetic than in non-diabetic patients, this is consistent with the finding of Rha and coworkers [33].

Diabetic patients have a great atherosclerotic burden, restenosis after PCI, and more adverse events probably because they are characterized by frequent hyperplasia after PCI, more easily activated platelets, increased levels of fibrinogen, thrombin and coagulation factor VII, proinflammatory states, systemic endothelial dysfunction, and metabolic disorders [21, 34, 35]. Of note, collateral circulation development is known to be less in diabetic patients than in non-diabetic patients when coronary arteries become occluded. Particularly, in CTO patients, well-developed coronary collateral circulation potentially supplies the downstream perfusion area and thereby alleviates myocardial ischemia, preserves viable myocardium, reduces infarct area, improves left ventricular function, and decrease cardiovascular mortality [36]. This may explain the worse outcome of CTO patients with DM [36].

Notably, previous cohort studies mainly focused on the outcomes of successful PCI as opposed to failed procedures in CTO patients with DM, thus reported different results [29, 30,37]. However, the higher rates of procedural complications and adverse events directly associated with failed CTO procedures were rarely considered thereby contributes to the poor prognosis of CTO patients [38]. The high rate of crossovers of failed CTO-PCI and medial therapy groups limits conclusions and may underestimate the actual effect of successful CTO-PCI. Further, patients treated via medical therapy without an attempted CTO-PCI were not enrolled in the previous studies [15]. Limited reports exist on the definite evidence of improved clinical outcomes of successful CTO-PCI compared with medical treatment (CTO-PCI not attempted), and the data is urgently needed $[15,16]$. Also, in the DECISION-CTO [39] and the Euro-CTO trials [40], detailed clinical outcomes of CTO patients with DM were not analyzed. Therefore, our study excluded patients who underwent failed CTO-PCI and rather investigated the clinical outcomes between successful CTO PCI and medical therapy (CTO-PCI not attempted) groups in CTO patients with and without
DM. Accordingly, our study is closer to the "real world" of the clinical practice in CTO patients with and without DM compared to previous studies.

Currently, there are no widely recognized consensus or guidelines on the treatment strategy of CTO patients with DM. Also, the prognosis of successful CTO-PCI versus medical therapy in this population is unknown. Contrary to the previous findings [22], our study showed that successful CTO-PCI reduces MACE compared to MT alone in CTO patients with DM. Nevertheless, among CTO patients without DM, we did not observe a reduction in MACE or cardiovascular mortality when compared with MT alone (although the MACE rate was higher in MT group). In addition, we performed PSM to adjust for potential selection bias and the influence of confounding factors, and maintain a balance in covariates. Results concurred with earlier findings before PSM was conducted. Besides, an ITT analysis of medical therapy versus initial CTO-PCI was performed for a highly comprehensive evaluation. Of note, initial CTO-PCI was highly beneficial to diabetic patients considering MACE when compared with MT. In the randomized COURAGE trial which compared PCI with MT in patients with stable coronary heart disease, subgroup analysis did not show any beneficial clinical outcomes among nondiabetic patients [41], this concurs with our findings. Additionally, we observed a significant interaction between diabetic or non-diabetic patients and therapeutic strategy regarding MACE, this suggests that the superiority of successful CTO-PCI over MT is dependent on the glucose level.

Besides, diabetic patients who are a higher- risk group were less likely to undergo CTO-PCI compared with non-diabetic patients. However, these higher-risk patients, highly benefit from the "treatment-risk paradox", which is a common procedure in PCI [21, 42]. Higher event rates in the high-risk subjects increase the statistical power in detecting the significant differences in adverse outcomes. These findings indicate that successful CTO-PCI has more clinical benefits in diabetic patients compared to non-diabetic patients. Besides, complete revascularization is associated with fewer MACEs and improved long-term survival in patients with multivessel disease and STEMI or angina [43, 44]. Previously, studies showed that success rates of CTO-PCI are similar in diabetic and non-diabetic patients [29, 30]. Furthermore, the two recent large CTO studies (OPEN CTO registry and PROGRESS CTO registry) in which current dedicated equipment and skills including hybrid algorithms have been applied, represent modern CTO-PCI standards. The two studies reported that high procedural success, however, similar and complication rates are low in patients with and without diabetes [24, 25]. Therefore, 
with the latest refinement equipment and techniques, PCI of CTO is safe, has high success rates, and poses low complication rates in patients with DM. In treating CTO patients with diabetes, CTO-PCI may be highly preferred as the treatment option.

\section{Study limitations}

This study is observational, though, we performed PSM to adjust the potential selection bias and minimize the confounding factors. Nevertheless, diabetes is on the rise as one of the leading causes of cardiovascular mortality worldwide, therefore, our findings on the highrisk subset of patients may be particularly meaningful.

\section{Conclusions}

Successful CTO-PCI potentially reduces the risk of MACE in diabetic patients compared to when medical therapy is used alone for treating chronic total occlusions. However, this intervention does not work for non-diabetic patients. Therefore, CTO-PCI provides a safe and effective treatment option for unselected CTO patients with diabetes. Large randomized clinical trials are thus warranted to verify these findings.

\section{Supplementary information}

Supplementary information accompanies this paper at https://doi. org/10.1186/s12933-020-01087-4.

Additional file 1: Table S1. Baseline clinical, angiographic and procedural characteristics of patients with and without diabetes stratified according to medical therapy or initial CTO-PCI. Table S2. Clinical outcomes of patients with and without diabetes stratified according to medical therapy or initial CTO-PCI.

\section{Abbreviations}

CABG: Coronary artery bypass grafting; CAD: Coronary artery disease; CTO: Chronic total occlusion; DM: Diabetes mellitus; J-CTO: Japanese-chronic total occlusion; LAD: Left anterior descending artery; LVEF: Left ventricular ejection fraction; MACE: Major adverse cardiovascular events; MI: Myocardial infarction; MT: Medical therapy; PCI: Percutaneous coronary intervention; PSM: Propensity score matching; TVR: Target-vessel revascularization.

\section{Acknowledgements}

We are grateful to Ying Liu and Kun Chen for their invaluable assistance.

\section{Authors' contributions}

LG was involved in the study design and manuscript drafting, and provided feedback on the manuscript. JJW, HYD, SKM, XYZ, HCL, JW, LZ and RCH helped to draft the manuscript. LG, HYD, SKM, JW and LZ collected data for this study. LG, JYX and XCZ conducted statistical analysis. JJW, HYD, HCL and RCH researched data and contributed to discussion. JJW, HYD and SKM helped to draft the revised manuscript. All authors read and approved the final manuscript.

\section{Funding}

This research did not receive any specific grant from funding agencies in the public, commercial, or not-for-profit sectors.

\section{Availability of data and materials}

The datasets generated and analyzed for this current study are available from the corresponding author upon reasonable request.

\section{Ethics approval and consent to participate}

The present study was approved by the First Affiliated Hospital of Dalian Medical University. Informed consent was exempt by the committee.

\section{Consent for publication}

All authors consent this manuscript for publication.

\section{Competing interests}

The authors declare that they have no competing interests.

\section{Author details}

${ }^{1}$ Department of Cardiology, The First Affiliated Hospital of Dalian Medical University, Dalian, People's Republic of China. ${ }^{2}$ Department of Radiology, Fuyang Hospital of Anhui Medical University, Fuyang, People's Republic of China.

${ }^{3}$ Department of Cardiology, Capital Medical University Affiliated Beijing

Friendship Hospital, Beijing, People's Republic of China.

Received: 12 May 2020 Accepted: 2 July 2020

Published online: 04 July 2020

\section{References}

1. Wild S, Roglic G, Green A, Sicree R, King H. Global prevalence of diabetes: estimates for the year 2000 and projections for 2030. Diabetes Care. 2004;27:1047-53.

2. Luscher TF, Creager MA, Beckman JA, Cosentino F. Diabetes and vascular disease: pathophysiology, clinical consequences, and medical therapy: part II. Circulation. 2003;108:1655-61.

3. Ruiz-Garcia J, Teles R, Rumoroso JR, et al. Comparison between diabetic and non-diabetic patients after successful percutaneous coronary intervention for chronic total occlusions in the drug-eluting stent era. Rev Port Cardiol. 2015;34:263-70.

4. Fefer P, Knudtson ML, Cheema AN, et al. Current perspectives on coronary chronic total occlusions: the Canadian Multicenter Chronic Total Occlusions registry. J Am Coll Cardiol. 2012;59:991-7.

5. Grantham JA, Marso SP, Spertus J, House J, Holmes DR, Rutherford BD. Chronic total occlusion angioplasty in the united states. JACC CardiovasC Interv. 2009;2:479-86

6. Sirnes PA, Myreng Y, Mølstad P, et al. Improvement in left ventricular ejection fraction and wall motion after successful recanalization of chronic coronary occlusions. Eur Heart J. 1998;19:273-81.

7. George S, Cockburn J, Clayton TC, et al. Long-term follow-up of elective chronic total coronary occlusion angioplasty: analysis from the U.K. Central Cardiac Audit Database. J Am Coll Cardiol. 2014;64:235-43.

8. Guo L, Wu J, Zhong L, et al. Two-year clinical outcomes of medical therapy vs. revascularization for patients with coronary chronic total occlusion. Hellenic J Cardiol. 2019. https://doi.org/10.1016/j.hjc.2019.03.006 (Epub ahead of print)

9. Yan Y, Zhang M, Yuan F, et al. Successful revascularization versus medical therapy in diabetic patients with stable right coronary artery chronic total occlusion: a retrospective cohort study. Cardiovasc Diabetol. 2019;18:108.

10. Rathore S, Matsuo H, Terashima M, et al. Procedural and in-hospital outcomes after percutaneous coronary intervention for chronic total occlusions of coronary arteries 2002 to 2008: impact of novel guidewire techniques. JACC Cardiovasc Interv. 2009;2:489-97.

11. Ladwiniec A, Allgar $\bigvee$, Thackray S, Alamgir F, Hoye A. Medical therapy, percutaneous coronary intervention and prognosis in patients with chronic total occlusions. Heart. 2015;101:1907-14.

12. Guo L, Lv HC, Zhong L, et al. Comparison of long-term outcomes of medical therapy and successful recanalisation for coronary chronic total occlusions in elderly patients: a report of 1,294 patients. Cardiovasc Diagn Ther. 2019;9:586-95.

13. Tomasello SD, Boukhris M, Giubilato S, et al. Management strategies in patients affected by chronic total occlusions: results from the Italian Registry of Chronic Total Occlusions. Eur Heart J. 2015;36:3189-98. 
14. Brilakis ES, Banerjee S, Karmpaliotis D, et al. Procedural outcomes of chronic total occlusion percutaneous coronary intervention: a report from the NCDR (National CardiovascularData Registry). JACC CardiovasC Interv. 2015;8:245-53.

15. Godino C, Bassanelli G, Economou Fl, et al. Predictors of cardiac death in patients with coronary chronic total occlusion not revascularized by $\mathrm{PCI}$. Int J Cardiol. 2013;168:1402-9.

16. Guo L, Zhang SF, Wu J, et al. Successful recanalisation of coronary chronic total occlusions is not associated with improved cardiovascular survival compared with initial medical therapy. Scand Cardiovasc J. 2019:53:305-11.

17. Guo L, Lv HC, Zhong L, et al. Gender differences in long-term outcomes of medical therapy and successful percutaneous coronary intervention for coronary chronic total occlusions. J Interv Cardiol. 2019. https://doi. org/10.1155/2019/2017958 (Epub ahead of print).

18. American Diabetes Association. Standards of Medical Care in Diabetes-2016: summary of revisions. Diabetes Care. 2016;39(Suppl 1):S4-5.

19. Guo L, Zhong L, Chen K, Wu J, Huang RC. Long-term clinical outcomes of optimal medical therapy vs. successful percutaneous coronary intervention for patients with coronary chronic total occlusions. Hellenic J Cardiol. 2018;59:281-7.

20. Cutlip DE, Windecker S, Mehran R, et al. Clinical end points in coronary stent trials: a case for standardized definitions: a case for standardized definitions. Circulation. 2007;115:2344-51.

21. Zhou M, Liu J, Hao Y, et al. Prevalence and in-hospital outcomes of diabetes among patients with acute coronary syndrome in China: findings from the Improving Care for Cardiovascular Disease in China-Acute Coronary Syndrome Project. Cardiovasc Diabetol. 2018;17:147.

22. Choi $\mathrm{KH}$, Yang JH, Song YB, et al. Long-term clinical outcomes of patients with coronary chronic total occlusion treated with percutaneous coronary intervention versus medical therapy according to presence of diabetes mellitus. Eurolntervention. 2017;13:970-7.

23. Claessen BE, Hoebers LP, van der Schaaf RJ, et al. Prevalence and impact of a chronic total occlusion in a non-infarct-related artery on long-term mortality in diabetic patients with ST elevation myocardial infarction. Heart. 2010;96:1968-72.

24. Martinez-Parachini JR, Karatasakis A, Karmpaliotis D, et al. Impact of diabetes mellitus on acute outcomes of percutaneous coronary intervention in chronic total occlusions: insights from a US multicentre registry. Diabetic Med. 2017;34:558-62.

25. Salisbury AC, Sapontis J, Grantham JA, et al. Outcomes of chronic total occlusion percutaneous coronary intervention in patients with diabetes: insights from the OPEN CTO registry. JACC Cardiovasc Interv. 2017;10:2174-81.

26. Qin Z, Zhou K, Li YP, et al. Remnant lipoproteins play an important role of in-stent restenosis in type 2 diabetes undergoing percutaneous coronary intervention: a single-centre observational cohort study. Cardiovasc Diabetol. 2019;18:11.

27. Seabra-Gomes R. Percutaneous coronary interventions with drug eluting stents for diabetic patients. Heart. 2006;92:410-9.

28. Kandzari DE, Rao SV, Moses JW, et al. Clinical and angiographic outcomes with sirolimus-eluting stents in total coronary occlusions: the ACROSS/ TOSCA-4 (Approaches to Chronic Occlusions With Sirolimus-Eluting Stents/Total Occlusion Study of Coronary Arteries-4) trial. JACC Cardiovasc Interv. 2009;2:97-106.

29. Safley DM, House JA, Rutherford BD, Marso SP. Success rates of percutaneous coronary intervention of chronic total occlusions and long-term survival in patients with diabetes mellitus. Diabetes Vasc Dis Res. 2006;3:45-51.
30. Claessen BE, Dangas GD, Godino C, et al. Long-term clinical outcomes of percutaneous coronary intervention for chronic total occlusions in patients with versus without diabetes mellitus. Am J Cardiol. 2011;108:924-31.

31. Pell JP, Pell AC, Jeffrey RR, et al. Comparison of survival following coronary artery bypass grafting vs. percutaneous coronary intervention in diabetic and non-diabetic patients: retrospective cohort study of 6320 procedures. Diabetic Med. 2004;21:790-2.

32. Wang Q, Liu H, Ding J. Outcomes of percutaneous coronary intervention in patients with coronary chronic total occlusions with versus without type 2 diabetes mellitus: a systematic review and meta-analysis. Medicine. 2017;96:e8499.

33. Rha SW, Choi CU, Na JO, et al. Comparison of 12-month clinical outcomes in diabetic and nondiabetic patients with chronic total occlusion lesions: a multicenter study. Coron Artery Dis. 2015;26:699-705.

34. Davi G, Catalano I, Averna M, et al. Thromboxane biosynthesis and platelet function in type II diabetes mellitus. N Engl J Med. 1990;322:1769-74.

35. Schofield I, Malik R, Izzard A, Austin C, Heagerty A. Vascular structural and functional changes in type 2 diabetes mellitus: evidence for the roles of abnormal myogenic responsiveness and dyslipidemia. Circulation. 2002;106:3037-43.

36. Shen Y, Ding FH, Dai Y, et al. Reduced coronary collateralization in type 2 diabetic patients with chronic total occlusion. Cardiovasc Diabetol. 2018;17:26.

37. Sanguineti F, Garot P, O'Connor S, et al. Chronic total coronary occlusion treated by percutaneous coronary intervention: long-term outcome in patients with and without diabetes. Eurolntervention. 2017;12:e1889-97.

38. Choo EH, Koh YS, Seo SM, et al. Comparison of successful percutaneous coronary intervention versus optimal medical therapy in patients with coronary chronic total occlusion. J Cardiol. 2019;73:156-62.

39. Lee SW, Lee PH, Ahn JM, et al. Randomized trial evaluating percutaneous coronary intervention for the treatment of chronic total occlusion. Circulation. 2019;139:1674-83.

40. Werner GS, Martin-Yuste V, Hildick-Smith D, et al. A randomized multicentre trial to compare revascularization with optimal medical therapy for the treatment of chronic total coronary occlusions. Eur Heart J. 2018;39:2484-93.

41. Boden WE, O'Rourke RA, Teo KK, et al. Optimal medical therapy with or without PCI for stable coronary disease. N Engl J Med. 2007;356:1503-16.

42. Iglesias JF, Degrauwe S, Rigamonti F, Noble S, Roffi M. Percutaneous coronary intervention of chronic total occlusions in patients with diabetes mellitus: a treatment-risk paradox. Curr Cardiol Rep. 2019;21:9.

43. Cui K, Lyu S, Liu H, et al. Staged complete revascularization or culpritonly percutaneous coronary intervention for multivessel coronary artery disease in patients with ST-segment elevation myocardial infarction and diabetes. Cardiovasc Diabetol. 2019;18:119.

44. Mancini GBJ, Boden WE, Brooks MM, et al. Impact of treatment strategies on outcomes in patients with stable coronary artery disease and type 2 diabetes mellitus according to presenting angina severity: a pooled analysis of three federally-funded randomized trials. Atherosclerosis. 2018;277:186-94.

\section{Publisher's Note}

Springer Nature remains neutral with regard to jurisdictional claims in published maps and institutional affiliations. 